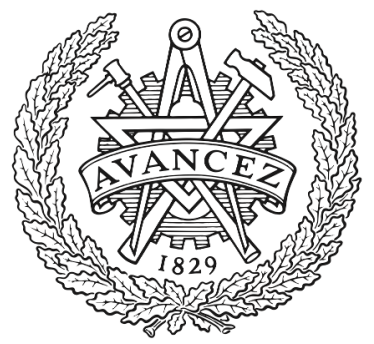

CHALMERS

UNIVERSITY OF TECHNOLOGY

\title{
ALMA Reveals a Gas-rich, Maximum Starburst in the Hyperluminous, Dust-obscured Quasar W0533-3401 at z similar to 2.9
}

Downloaded from: https://research.chalmers.se, 2023-04-26 11:56 UTC

Citation for the original published paper (version of record):

Fan, L., Knudsen, K., Han, Y. et al (2019). ALMA Reveals a Gas-rich, Maximum Starburst in the Hyperluminous, Dust-obscured Quasar W0533-3401 at z similar to 2.9. Astrophysical Journal, 887(1). http://dx.doi.org/10.3847/1538-4357/ab5059

N.B. When citing this work, cite the original published paper. 


\title{
ALMA Reveals a Gas-rich, Maximum Starburst in the Hyperluminous, Dust-obscured Quasar W0533-3401 at $z \sim 2.9$
}

\author{
Lulu Fan (范璐璐) $)^{1,2,3}$ (D), Kirsten K. Knudsen ${ }^{4}$ (D), Yunkun Han (韩云坤) $)^{5,6,7}$ (D), and Qing-hua Tan (谈清华) $)^{8}$ (D) \\ ${ }^{1}$ CAS Key Laboratory for Research in Galaxies and Cosmology, Department of Astronomy, University of Science and Technology of China, Hefei 230026, People's \\ Republic of China; 1lfan@ustc.edu.cn \\ ${ }^{2}$ School of Astronomy and Space Sciences, University of Science and Technology of China, Hefei, Anhui 230026, People's Republic of China \\ ${ }^{3}$ Shandong Provincial Key Lab of Optical Astronomy and Solar-Terrestrial Environment, Institute of Space Science, Shandong University, Weihai, 264209, People's \\ Republic of China \\ ${ }_{5}^{4}$ Department of Space, Earth and Environment, Chalmers University of Technology, Onsala Space Observatory, SE-439 92 Onsala, Sweden \\ ${ }^{5}$ Yunnan Observatories, Chinese Academy of Sciences, 396 Yangfangwang, Guandu District, Kunming, 650216, People's Republic of China \\ ${ }^{6}$ Center for Astronomical Mega-Science, Chinese Academy of Sciences, 20A Datun Road, Chaoyang District, Beijing, 100012, People's Republic of China \\ ${ }^{7}$ Key Laboratory for the Structure and Evolution of Celestial Objects, Chinese Academy of Sciences, 396 Yangfangwang, Guandu District, Kunming, 650216, \\ People's Republic of China \\ ${ }^{8}$ Purple Mountain Observatory \& Key Laboratory for Radio Astronomy, Chinese Academy of Sciences, 10 Yuanhua Road, Nanjing 210033, People's Republic of \\ China \\ Received 2019 April 14; revised 2019 October 2; accepted 2019 October 21; published 2019 December 11
}

\begin{abstract}
We present ALMA observations and multiwavelength spectral energy distribution analysis in a Wide-field Infrared Survey Explorer-selected, hyperluminous dust-obscured quasar W0533-3401 at $z=2.9$. We derive the physical properties of each of its components, such as molecular gas, stars, dust, and the central supermassive black hole (SMBH). Both the dust continuum at $3 \mathrm{~mm}$ and the $\mathrm{CO}(3-2)$ line are detected. The derived molecular gas mass $M_{\text {gas }}=8.4 \times 10^{10} M_{\odot}$ and its fraction $f_{\text {gas }}=0.7$ suggest that W0533-3401 is gas-rich. The star formation rate (SFR) has been estimated to be $\sim 3000-7000 M_{\odot} \mathrm{yr}^{-1}$ by using different methods. The high values of SFR and specific SFR suggest that W0533-3401 is a maximum starburst. The corresponding gas depletion timescales are very short $\left(t_{\mathrm{depl}} \sim 12-28 \mathrm{Myr}\right)$. The $\mathrm{CO}(3-2)$ emission line is marginally resolved and has a velocity gradient, which is possibly due to a rotating gas disk, gas outflow, or merger. Finally, we infer the black hole mass growth rate of W0533-3401 $\left(\dot{M}_{\mathrm{BH}}=49 M_{\odot} \mathrm{yr}^{-1}\right)$, which suggests a rapid growth of the central SMBH. The observed black hole to stellar mass ratio $M_{\mathrm{BH}} / M_{\star}$ of W0533-3401, which is dependent on the adopted Eddington ratio, is over one order of magnitude higher than the local value, and is evolving toward the evolutionary trend of unobscured quasars. Our results are consistent with the scenario that W0533-3401, with both a gas-rich maximum starburst and a rapid black hole growth, is experiencing a short transition phase toward an unobscured quasar.
\end{abstract}

Unified Astronomy Thesaurus concepts: Active galaxies (17); High-redshift galaxies (734); Starburst galaxies (1570); Galaxy evolution (594); Quasars (1319)

\section{Introduction}

Supermassive black holes (SMBH) have been discovered in the centers of local elliptical galaxies, and the stellar bulge mass in galaxies is found to be correlated with the mass of their central SMBHs (e.g., Magorrian et al. 1998; Ferrarese \& Ford 2005), implying that galaxies and their central SMBHs could coevolve (Kormendy \& Ho 2013). In the popular framework of massive galaxy formation and coevolution with the central SMBH (e.g., Hopkins et al. 2008), galaxy gas-rich mergers trigger intense starbursts and also provide the fuel for central SMBH accretion. An evolutionary sequence is predicted that the evolution of massive galaxies will experience several phases: starburst, dust-obscured quasar, unobscured quasar and finally a passively evolved galaxy (e.g., Sanders et al. 1988; Granato et al. 2004; Alexander \& Hickox 2012). Dust-obscured quasars have been believed to represent a brief transition phase linking intense starbursts and unobscured quasars, and will be good candidates for studying the interplay between host galaxies and their central SMBHs (e.g., Hickox \& Alexander 2018).

Among several selection techniques commonly used to identify and characterize obscured quasars (see a recent review by Hickox \& Alexander 2018), the mid-IR color-color diagnostics (Padovani et al. 2017) are efficient and effective for identifying heavily dust-obscured, powerful quasars. Recently, a new population of luminous, dust-obscured galaxies has been discovered based on a so-called W1W2-dropout color-selected method (Eisenhardt et al. 2012; Wu et al. 2012) which uses only four mid-IR wavebands of the Wide-field Infrared Survey Explorer (WISE; Wright et al. 2010) all-sky survey. Follow-up studies, including UV/optical spectral analysis (Wu et al. 2012, 2018), IR spectral energy distribution (SED) analysis (Tsai et al. 2015; Fan et al. 2016, 2018), X-ray observations (Stern et al. 2014; Piconcelli et al. 2015; Assef et al. 2016; Ricci et al. 2017; Vito et al. 2018; Zappacosta et al. 2018), and high-resolution radio imaging (Frey et al. 2016), suggest that these WISE-selected galaxies are mainly powered by accreting SMBHs and substantially dust-obscured quasars.

Multiwavelength observations have been carried out to investigate the physical properties of each component in these dust-obscured quasars, such as stars, dust, gas, the central SMBH, galaxy morphology, and the environment they reside in (e.g., Wu et al. 2014; Assef et al. 2015; Jones et al. 2015, 2017; Díaz-Santos et al. 2016, 2018; Fan et al. 2016, 2017, 2018; Tsai et al. 2018). All results are generally consistent with the merger-driven SMBH-host coevolution scenario.

In this paper, we report the results of ALMA observations and a thorough UV-to-millimeter SED analysis of a WISE-selected 
dust-obscured quasar W0533-3401 at $z \sim 2.9$, which is among the most luminous obscured quasars with a total IR luminosity greater than $10^{14} L_{\odot}$ (Tsai et al. 2015). Our previous study based on IR SED decomposition suggests that W0533-3401 has simultaneously rapid growth of SMBH and intense starburst $\left(>3000 M_{\odot} \mathrm{yr}^{-1}\right)$ (Fan et al. 2016). With the present ALMA observations and multiwavelength SED analysis, we can further derive the properties of each component in the host galaxy and explore the potential relation between the assembly of host galaxy and the central SMBH accretion. In Section 2, we present our ALMA observations and data analysis on W0533 -3401. In Section 3, we compile the multiwavelength data and introduce our SED modeling method. We show our main results and discussion in Section 4. In Section 5, we summarize our conclusions. Throughout this work we assume a standard, flat $\Lambda$ CDM cosmology (see Komatsu et al. 2011), with $H_{0}=$ $70 \mathrm{~km} \mathrm{~s}^{-1}, \Omega_{M}=0.3$, and $\Omega_{\Lambda}=0.7$.

\section{ALMA Observations and Data Analysis}

Observations of W0533-3401 were obtained with ALMA using the band-3 receiver as a part of project 2017.1.00441.S. The observations were carried out on 2017 December 20 using 45 antennas in a configuration with baseline length ranging from 15 to $2460 \mathrm{~m}$. The on-source integration time was $517 \mathrm{~s}$. The sources J0538-4405 and J0522-3627 were used for bandpass and flux calibration, and for gain calibration, respectively. The uncertainty on the absolute flux calibration was estimated to be about $5 \%$. The precipitable water vapor was measured to be $4.2-4.5 \mathrm{~mm}$ and the weather conditions were stable during this relatively short period. The receiver settings were used as follows: the lower sideband has two spectral windows tuned to 87.418 and $89.219 \mathrm{GHz}$ with spectral line mode using 960 spectral channels each, where the tuning was selected to target $\mathrm{CO}(3-2)$ using the optical redshift $z_{\text {opt }}=2.904$ (Tsai et al. 2015), and the upper sideband has two spectral windows tuned to 99.421 and $101.221 \mathrm{GHz}$ in continuum mode with 128 channels each.

The data were processed using CASA (Common Astronomy Software Application ${ }^{9}$; McMullin et al. 2007). We checked the data calibration from observatory delivered pipeline processing. We found that the calibration was sufficient and made no further adjustments. The calibrated visibilities were reimaged using task tclean. For natural weighting, the angular resolution of the observations (clean beam size) is 0 " $61 \times$ 0 ".53, at a position angle (P.A.) $=-78^{\circ} .4$, and the $\mathrm{rms}$ is $0.43 \mathrm{mJy} /$ beam in $53 \mathrm{~km} \mathrm{~s}^{-1}$ channels. A summary of target properties and ALMA measurements can be found in Table 1.

\section{Multiwavelength Data and SED Modeling}

\subsection{UV-to-millimeter SED}

We construct the multiwavelength SED of W0533-3401 by compiling the optical to millimeter broadband photometry from various catalogs available in the literature (see Table 2). Optical/near-infrared photometry in five broad bands, grizY, are retrieved from the first public data release of the Dark Energy Survey (DES DR1; Abbott et al. 2018). ${ }^{10}$ Near-infrared $J$ band photometry has been obtained by SOAR/OSIRIS (Assef et al. 2015). The WISE W3 and W4 photometry

\footnotetext{
9 https://casa.nrao.edu/

${ }^{10}$ https://des.ncsa.illinois.edu/releases/dr1/
}

Table 1

Summary of Target Properties and ALMA Measurements

\begin{tabular}{|c|c|}
\hline $\begin{array}{l}\text { Name } \\
\text { R.A. WISE (J2000) } \\
\text { Decl. WISE }(\mathrm{J} 2000) \\
z_{\text {opt }}\end{array}$ & $\begin{array}{c}\text { W0533-3401 } \\
05: 33: 58.44 \\
-34: 01: 34.5 \\
2.904\end{array}$ \\
\hline Date of ALMA observations & 2017 Dec 20 \\
\hline Number of antennas & 45 \\
\hline R.A.CO(3-2) (J2000) & $05: 33: 58.42$ \\
\hline $\operatorname{Dec}_{\mathrm{CO}(3-2)}(\mathrm{J} 2000)$ & $-34: 01: 34.5$ \\
\hline$z_{\mathrm{CO}(3-2)}$ & $2.9026 \pm 0.0003$ \\
\hline $\operatorname{Size}_{\mathrm{CO}(3-2)}\left(\operatorname{arcsec}^{2}\right)$ & $(0.73 \pm 0.14) \times(0.37 \pm 0.14)$ \\
\hline P.A.CO(3-2) (deg) & $126 \pm 18$ \\
\hline FWHM $\left(\mathrm{km} \mathrm{s}^{-1}\right)^{\mathrm{a}}$ & $566 \pm 44$ \\
\hline$I_{\mathrm{CO}(3-2)}\left(\mathrm{Jy} \mathrm{km} \mathrm{s}^{-1}\right)$ & $2.01 \pm 0.13$ \\
\hline$L_{\mathrm{CO}(3-2)}^{\prime}\left(10^{10} \mathrm{~K} \mathrm{~km} \mathrm{~s}^{-1} \mathrm{pc}^{2}\right)$ & $8.4 \pm 0.5$ \\
\hline$S_{3 \mathrm{~mm}}[\mathrm{mJy}]$ & $0.140 \pm 0.033$ \\
\hline
\end{tabular}

Table 2

Multiwavelength Photometry Data

\begin{tabular}{lccc}
\hline \hline Band & $\begin{array}{c}\text { Wavelength } \\
(\mu \mathrm{m})\end{array}$ & $\begin{array}{c}\text { Frequency } \\
(\mathrm{GHz})\end{array}$ & $\begin{array}{c}\text { Flux Density } \\
(\mathrm{mJy})\end{array}$ \\
\hline CTIO/DECam $g$ & 0.36 & 833333 & $0.0052 \pm 0.0002$ \\
CTIO/DECam $r$ & 0.54 & 555556 & $0.0075 \pm 0.0003$ \\
CTIO/DECam $i$ & 0.64 & 468750 & $0.0099 \pm 0.0005$ \\
CTIO/DECam $z$ & 0.77 & 389610 & $0.0135 \pm 0.0011$ \\
CTIO/DECam $Y$ & 0.90 & 333333 & $0.0119 \pm 0.0033$ \\
SOAR/OSIRIS $J$ & 1.05 & 285714 & $0.0128 \pm 0.0013$ \\
WISE/W1 & 3.4 & 88174.2 & $0.0351 \pm 0.0016$ \\
WISE/W2 & 4.6 & 65172.3 & $0.0726 \pm 0.0036$ \\
WISE/W3 & 12 & 24982.7 & $3.0 \pm 0.1$ \\
WISE/W4 & 22 & 13626.9 & $11.9 \pm 1.1$ \\
Herschel/PACS & 70 & 4143.65 & $39.3 \pm 5.9$ \\
Herschel/PACS & 160 & 1805.05 & $97.4 \pm 14.0$ \\
Herschel/SPIRE & 250 & 1199.17 & $107.5 \pm 4.8$ \\
Herschel/SPIRE & 350 & 856.55 & $76.3 \pm 7.3$ \\
Herschel/SPIRE & 500 & 599.585 & $48.9 \pm 4.5$ \\
ALMA & 3000 & 100.00 & $0.140 \pm 0.033$ \\
\hline
\end{tabular}

of W0533-3401 are from the ALLWISE Data Release (Cutri 2013). W3 and W4 flux densities and uncertainties have been converted from catalog Vega magnitude by using zeropoints of 29.04 and 8.284 Jy, respectively (Wright et al. 2010). While for WISE $W 1$ and $W 2$ photometry, we do the aperture photometry based on the unblurred coadded WISE images ${ }^{11}$ (unWISE, Lang 2014; Meisner et al. 2017). The photometry errors have been estimated based on the inverse variance images. We also collect the FIR photometry of W0533-3401 obtained with Herschel (Pilbratt et al. 2010) PACS (Poglitsch et al. 2010) at 70 and $160 \mu \mathrm{m}$ and SPIRE (Griffin et al. 2010) at 250,350 , and $500 \mu \mathrm{m}$ in our previous work (Fan et al. 2016). Our ALMA observations show a marginal detection $(\sim 4.2 \sigma)$ of $3 \mathrm{~mm}$ dust continuum. The measured observedframe continuum flux density is $0.140 \pm 0.033 \mathrm{mJy}$ (see Figure 1).

11 https://unwise.me/ 


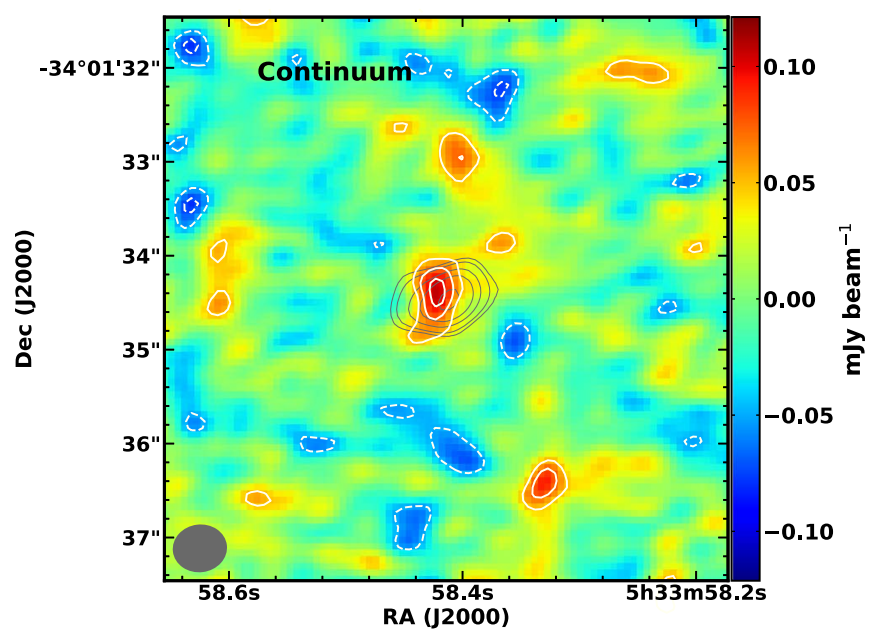

Figure 1. ALMA $3 \mathrm{~mm}$ dust continuum map of W0533-3401 imaged with natural weighting. The ivory-colored contours are at $-3,-2,2,3$, and $4 \sigma$ level. The beam size is shown in gray and is about $\sim 0$ !" $6 \times 0$." 5 . The gray contours represent the natural weighted $\mathrm{CO}(3-2)$ moment- 0 map at $4,5,7,9$, and $12 \sigma$ level (see Figure 3).

\subsection{Multiwavelength SED Analysis}

For the multiwavelength SED analysis of W0533-3401, we use a forthcoming version (Y. Han et al. 2019, in preparation) of the Bayesian SED modeling and interpreting code BAYESED ${ }^{12}$ (Han \& Han 2012, 2014, 2019). In the new version of BAYESED, the stellar emission, dust attenuation, and dust emission can be consistently connected by assuming an energy balance, a technique similar to that employed in MAGPHYS (da Cunha et al. 2008) and CIGALE (Noll et al. 2009; Boquien et al. 2019). The stellar emission is modeled by using the Bruzual \& Charlot (2003) SSP with a Chabrier (2003) initial mass function, an exponentially declining star formation history (SFH) and the Calzetti et al. (2000) dust attenuation law. The energy of stellar emission absorbed by dust is assumed to be totally re-emitted at the IR band, which is modeled by a graybody. The graybody model is defined as $S_{\lambda} \propto$ $\left(1-e^{-\left(\frac{\lambda_{0}}{\lambda}\right)^{\beta}}\right) B_{\lambda}\left(T_{\text {dust }}\right)$, where $B_{\lambda}$ is the Planck blackbody spectrum and $\lambda_{0}=125 \mu \mathrm{m}$. Dust temperature $T_{\text {dust }}$ and the emissivity index $\beta$ are two free parameters. Finally, as in Fan et al. (2016), the active galactic nucleus (AGN) torus emission is modeled independently with the extensive database ${ }^{13}$ of 1,247,400 SEDs from the CLUMPY torus model (Nenkova et al. 2008a, 2008b). A $k$-dimensional tree-based nearest-neighbor searching technique has been employed to allow us to evaluate the CLUMPY torus model at any point in its 6D parameter space. Note that the CLUMPY model includes not only the torus dust emission, but also a part of the AGN accretion disk emission that is scattered into our line of sight or not absorbed by torus dust. Thus the CLUMPY model can provide a consistent description of AGN UV-to-millimeter SED. In total, the three-component model, including stars, AGN, and cold dust emissions, has 12 free parameters. The priors for them are summarized in Table 3.

In Figure 2, we show the best-fit three-component SED model (black solid line) with BAYESED to the observed

\footnotetext{
12 https://bitbucket.org/hanyk/bayesed/

13 http://www.pa.uky.edu/clumpy/models/clumpy_models_201410_ tvavg.hdf5/
}

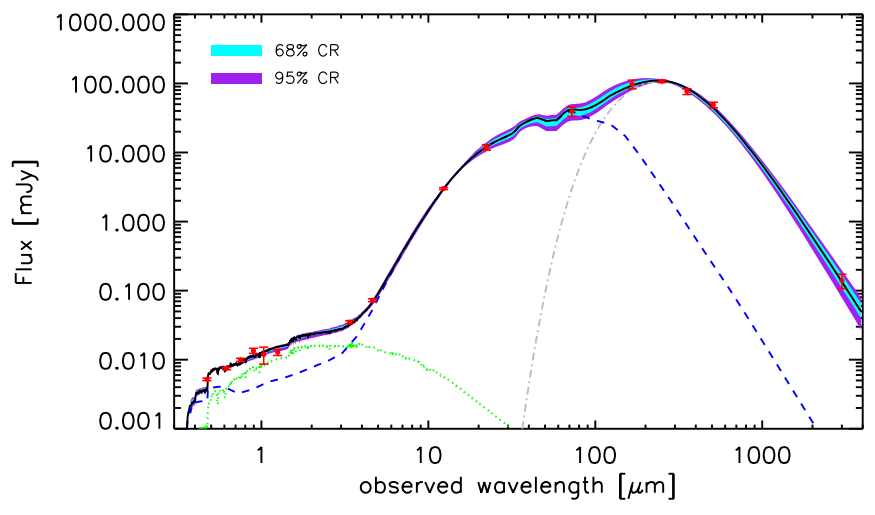

Figure 2. Best-fit three-component SED model (black solid line) with BAYESED to the observed UV-to-millimeter SED of W0533-3401 (red points). The color-filled regions with cyan and purple show the $68 \%$ and $95 \%$ CR of the best-fit model, respectively. The green dotted line, the blue dashed line, and the gray dotted-dashed line represent the emissions from stars, AGN, and cold dust, respectively.

Table 3

The Model Parameters, Their Priors and Best-fitting Quantities for Multiwavelength SED Analysis

\begin{tabular}{|c|c|c|c|c|}
\hline Name & Prior & Min & Max & $\begin{array}{l}\text { Best-fitting } \\
\text { Value }\end{array}$ \\
\hline \multicolumn{5}{|c|}{ Stellar component ${ }^{\mathrm{a}}$} \\
\hline $\log \left(\right.$ age $\left.\mathrm{yr}^{-1}\right)$ & $\begin{array}{l}\text { Uniform, } \\
\quad \text { age }<\operatorname{age}_{\mathrm{U}}(z)\end{array}$ & 5 & 10.3 & $6.7_{-0.1}^{+0.1}$ \\
\hline $\log \left(\tau \mathrm{yr}^{-1}\right)$ & Uniform & 6 & 12 & $9.1_{-1.9}^{+1.8}$ \\
\hline $\log \left(Z / Z_{\odot}\right)$ & Uniform & -2.3 & 0.7 & $0.28_{-0.38}^{+0.18}$ \\
\hline$A_{\mathrm{V}} / \mathrm{mag}$ & Uniform & 0 & 4 & $1.81_{-0.08}^{+0.08}$ \\
\hline \multicolumn{5}{|c|}{ Graybody model } \\
\hline$T_{\text {dust }} / \mathrm{K}$ & Uniform & 10 & 100 & $78.1_{-5.2}^{+5.9}$ \\
\hline$\beta$ & Uniform & 1 & 3 & $1.84_{-0.13}^{+0.14}$ \\
\hline \multicolumn{5}{|c|}{ CLUMPY model $^{\mathrm{b}}$} \\
\hline$N_{0}$ & Uniform & 1 & 15 & $5.9_{-1.0}^{+1.3}$ \\
\hline$Y$ & Uniform & 5 & 100 & $48.2_{-28.2}^{+32.6}$ \\
\hline$i$ & Uniform & 0 & 90 & $37.8_{-23.8}^{+20.0}$ \\
\hline$q$ & Uniform & 0 & 3 & $2.2_{-0.4}^{+0.4}$ \\
\hline$\sigma$ & Uniform & 15 & 70 & $62.3_{-5.1}^{+8.5}$ \\
\hline$\tau_{\mathrm{V}}$ & Uniform & 10 & 300 & $22.2_{-5.1}^{+5.4}$ \\
\hline
\end{tabular}

Notes.

${ }^{\text {a }}$ Four parameters of stellar components: age (age), the e-folding time for the exponentially declining SFH $(\tau)$, stellar metallicity $(Z)$, and dust attenuation $\left(A_{\mathrm{V}}\right)$.

${ }^{\mathrm{b}}$ The detailed description and definition of six free parameters for the CLUMPY model can be found in Nenkova et al. (2008a, 2008b).

UV-to-millimeter SED of W0533-3401 (red points). With a newly developed function in the new version of BAYESED, we can provide the confidence regions (CR) for our best-fit SED model. We plot the 68\% and 95\% CR with color-filled regions (cyan and purple, respectively). Absorbed stellar emission, AGN emission, and cold dust emission are shown by the green dotted line, the blue dashed line, and the gray dotted-dashed line, respectively. The derived properties have been shown in Table 4 and the results will be discussed in the next section. 
Table 4

Derived Properties of W0533-3401

\begin{tabular}{lccc}
\hline \hline Parameter & Value & Unit & Note \\
\hline$M_{\star}$ & $(3.5 \pm 0.9) \times 10^{10}$ & $M_{\odot}$ & $(1)$ \\
$\mathrm{SFR}$ & $6985 \pm 3006$ & $M_{\odot} \mathrm{yr}^{-1}$ & $(2)$ \\
$\mathrm{sSFR}$ & $200 \pm 100$ & $\mathrm{Gyr}^{-1}$ & $(3)$ \\
$L_{\mathrm{AGN}}$ & $(7.0 \pm 0.6) \times 10^{13}$ & $L_{\odot}$ & $(4)$ \\
$L_{\mathrm{GB}}$ & $(3.5 \pm 0.4) \times 10^{13}$ & $L_{\odot}$ & $(5)$ \\
$L_{\star}^{\text {unabs }}$ & $(3.6 \pm 0.4) \times 10^{13}$ & $L_{\odot}$ & $(6)$ \\
$L_{\star}^{\text {abs }}$ & $(9.7 \pm 1.0) \times 10^{11}$ & $L_{\odot}$ & $(7)$ \\
$M_{\mathrm{H}}$ & $(8.4 \pm 0.5) \times 10^{10}$ & $M_{\odot}$ & $(8)$ \\
$M_{\text {dyn }}$ & $1.6 \times 10^{11}$ & $M_{\odot}$ & $(9)$ \\
$M_{\mathrm{BH}}$ & $2.2 \times 10^{9}$ & $M_{\odot}$ & $(10)$ \\
$M_{\text {dust }}$ & $M_{\odot}$ & $(11)$ \\
$\mathrm{T}_{\mathrm{dust}}$ & $(8.9 \pm 1.6) \times 10^{7}$ & $\mathrm{~K}$ & $(12)$ \\
$\beta$ & $78.1 \pm 5.6$ & & $(13)$ \\
$\mathrm{A}_{\mathrm{V}}$ & $1.84 \pm 0.14$ & $\mathrm{mag}$ & $(14)$ \\
$t_{\text {depl }}$ & $1.81 \pm 0.08$ & $\mathrm{Myr}$ & $(15)$ \\
\hline
\end{tabular}

Note. (1): Stellar mass. (2): Star formation rate. (3): Specific star formation rate. (4): Bolometric luminosity of AGN component. (5): IR luminosity of cold dust. (6): Bolometric luminosity of attenuation-corrected stellar emission. (7): Bolometric luminosity of absorbed stellar emission. (8): Molecular gas mass. (9): Dynamical mass. (10): The central suppermassive black hole mass by assuming $\lambda_{\text {Edd }}=1$. (11): Dust mass. (12): Dust temperature. (13): Dust emissivity index. (14): Dust attenuation. (15): The gas depletion timescales.

\section{Results and Discussion}

\section{1. $\mathrm{CO}(3-2)$ Line Emission}

In Figures 3 and 4, we show the resulting line detection, both as spectrum and moment-maps. The $\mathrm{CO}$ (3-2) emission line peaks at a frequency corresponding to a redshift of $z_{\mathrm{CO}(3-2)}=$ $2.9026 \pm 0.0003$. The detected CO (3-2) line is spatially extended and shows a velocity gradient. We fit the velocityintegrated visibilities with an elliptical Gaussian function. The derived source size deconvolved from the beam is $(0.73 \pm 0.14) \times(0.37 \pm 0.14) \operatorname{arcsec}^{2}$ with P.A. $=126 \pm 18$ We measure the line flux $I_{\mathrm{CO}(3-2)}=2.01 \pm 0.13 \mathrm{Jy} \mathrm{km} \mathrm{s}^{-1}$. The rms is determined in the inner $10^{\prime \prime}$ of the moment map excluding the central part that contains the source itself. A single Gaussian fit to the continuum-subtracted $\mathrm{CO}$ (3-2) spectrum gives CO (3-2) FWHM $=566 \pm 44 \mathrm{~km} \mathrm{~s}^{-1}$. From the $\mathrm{CO}(3-2)$ line flux, we can derive the $\mathrm{CO}$ line luminosity $L_{\mathrm{CO}(3-2)}^{\prime}=(8.4 \pm 0.5) \times 10^{10} \mathrm{~K} \mathrm{~km} \mathrm{~s}^{-1} \mathrm{pc}^{2}$, by using Equation (3) in Solomon \& Vanden Bout (2005). The continuum is marginally detected, as shown in Figure 1. The extend of the continuum emission is smaller than that of the $\mathrm{CO}$ (3-2) line, which is likely due to a combination of the optical depth effects and the signal-to-noise ratio of continuum detection. The results are summarized in Table 1.

We can infer the molecular gas mass directly from the measured $\mathrm{CO}$ line luminosity. We adopt the excitation ratio between the $\mathrm{CO}(3-2)$ and $\mathrm{CO}(1-0)$ line of $r_{32 / 10}=0.8$ as suggested by Banerji et al. (2017). The adopted ratio is intermediate between the typical values for submillimeter galaxies $\left(\right.$ SMGs, $\left.r_{32 / 10}=0.66\right)$ and optical quasars $\left(r_{32 / 10}=\right.$ 0.97) from Carilli \& Walter (2013), and consistent with the expectation that obscured quasars represent the transition phase from SMGs to unobscured quasars. The calculated $\mathrm{CO}(1-0)$ line luminosity is $L_{\mathrm{CO}(1-0)}^{\prime}=(10.5 \pm 0.6) \times 10^{10} \mathrm{~K} \mathrm{~km} \mathrm{~s}^{-1} \mathrm{pc}^{2}$. We also adopt the CO-to- $\mathrm{H}_{2}$ conversion factor, $\alpha_{\mathrm{CO}}=$ $0.8 M_{\odot}\left(\mathrm{K} \mathrm{km} \mathrm{s}^{-1} \mathrm{pc}^{2}\right)^{-1}$, which is suggested to be appropriate for starbursts and quasar hosts (Carilli \& Walter 2013). Under these considerations, we obtain a molecular gas mass of $M_{\mathrm{H}_{2}}=$ $(8.4 \pm 0.5) \times 10^{10} M_{\odot}$.

We show the $\mathrm{CO}(3-2)$ velocity map in the right panel of Figure 3 and the position versus velocity (PV) diagram extracted along the major axis at P.A. $=330^{\circ}$ in Figure 5. The CO (3-2) emission line is marginally resolved. Both figures show the possible presence of a velocity gradient. Such a velocity gradient, which has also been observed in many other high-redshift quasars (e.g., Leung et al. 2017; Brusa et al. 2018; Feruglio et al. 2018; Talia et al. 2018), could be possibly the signature of a rotating disk of molecular gas, although the explanation is not unique. Especially, tentatively emission is seen at $\sim 3 \sigma$ level extending to the west side of the source (see the left panel of Figure 3). This suggests that the gas-rich latestage major merger and gas outflow are also possible for originating such a velocity gradient (e.g., Springel \& Hernquist 2005; Hopkins et al. 2009; Ueda et al. 2014; Hung et al. 2015). However, due to limited depth and resolution, we cannot make a solid conclusion. Deeper observation would be required to distinguish them.

Assuming that the gas is distributed in a rotating disk, we can investigate the kinematic properties of the molecular gas traced by the $\mathrm{CO}(3-2)$ line, using ${ }^{3 \mathrm{D}} \mathrm{BAROLO}$, a tool for fitting 3D tilted-ring models to emission-line data cubes (Di Teodoro \& Fraternali 2015). We assume a disk model with three rings and a ring width of 0.2 . We fix P.A. $=330^{\circ}$ and adopt an inclination of $59^{\circ} \pm 14^{\circ}$, which is inferred from the observed ratio of minor to major axis. The derived rotation velocity $\left(V_{\text {rot }}\right)$ and the intrinsic velocity dispersion $\sigma$ are about $\sim 240 \mathrm{~km} \mathrm{~s}^{-1}$ and $60 \mathrm{~km} \mathrm{~s}^{-1}$, respectively. The ratio of rotation velocity to velocity dispersion $V_{\text {rot }} / \sigma$ for W0533-3401 is about 4 , which is close to the typical value $\sim 7$ for the molecular gas in $z>1$ star-forming galaxies (Tacconi et al. 2013). The large ratio $V_{\text {rot }} / \sigma$ indicates that the molecular gas is turbulent, which is possibly due to a thick, dynamically hot disk in W0533-3401. A similar finding has been reported by Tadaki et al. (2018); they found a gravitationally unstable, rotating gas disk in an extreme starburst galaxy at $z \sim 4$. The dynamical mass within the CO-emitting region can be estimated by applying the relation in Wang et al. (2013), $M_{\text {dyn }} / M_{\odot}=1.16 \times 10^{5} \times$ $\left(0.75 \times \mathrm{FWHM}_{\mathrm{CO}}\right)^{2} \times D / \sin i$, where $D$ is the disk diameter in kiloparsecs from the CO (3-2) measurement and $i$ is the inclination angle. This gives $M_{\mathrm{dyn}}=1.6 \times 10^{11} M_{\odot}$.

\subsection{Dust Properties}

Cold dust emission heated by stars has been modeled with a graybody model in our multiwavelength SED analysis (see Section 3.2 and Figure 2). The best-fit model gives the estimations of dust properties: IR luminosity $L_{\mathrm{GB}}$, dust temperature $T_{\mathrm{dust}}$, and the emissivity index $\beta$, which are listed in Table 4. The derived values are consistent with those in our previous work based on only IR SED decomposition (Fan et al. 2016). Dust mass can also be calculated by using the following equation:

$$
M_{\mathrm{dust}}=\frac{D_{\mathrm{L}}^{2}}{(1+z)} \times \frac{S_{\nu_{\mathrm{obs}}}}{\kappa_{\nu_{\mathrm{rest}}} B\left(\nu_{\mathrm{rest}}, T_{\mathrm{dust}}\right)},
$$

where $D_{\mathrm{L}}$ is the luminosity distance, $S_{\nu_{\mathrm{obs}}}$ is the flux density at observed frequency $\nu_{\text {obs }}, \kappa_{\nu_{\text {rest }}}=\kappa_{0}\left(\nu / \nu_{0}\right)^{\beta}$ is the dust mass absorption coefficient at the rest frequency of the observed 

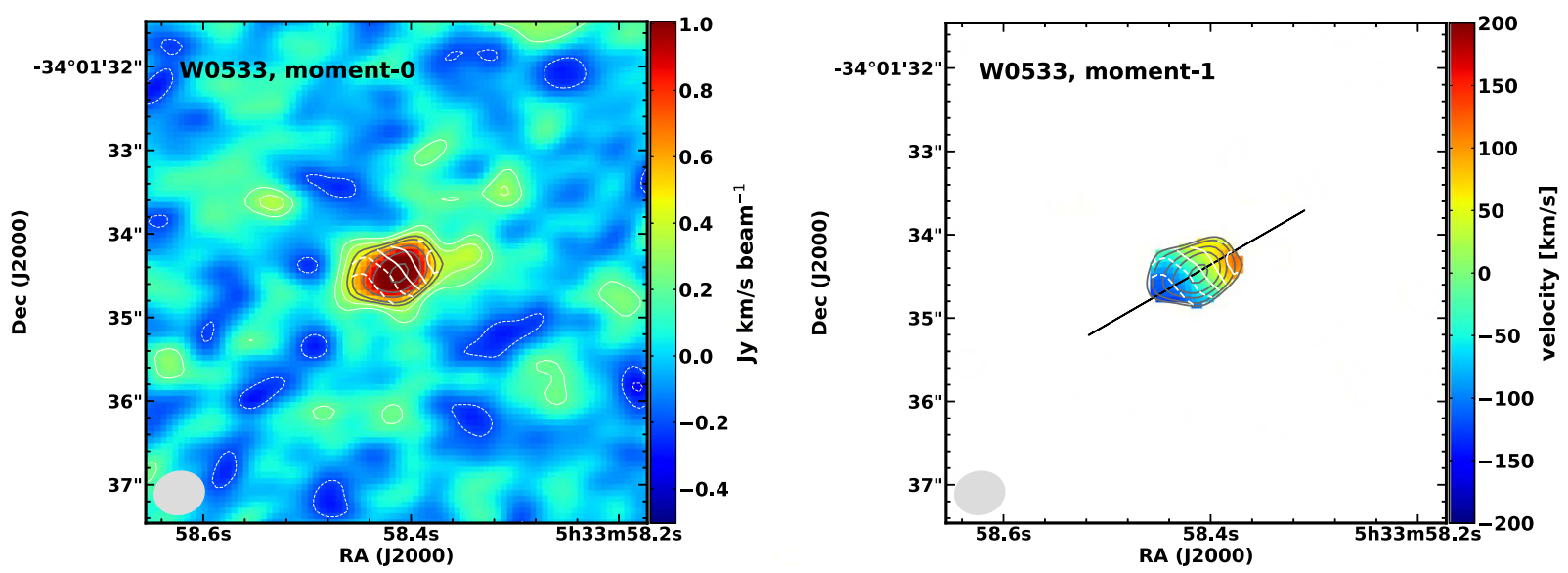

Figure 3. In the left panel, we plot a moment- 0 map of the $\mathrm{CO}(3-2)$ emission line, where the gray contours represent the moment- 0 at 4,5 , 7, 9, and $12 \sigma$ and the ivory contours are at $-3,-2,2$, and $3 \sigma$ levels $\left(\sigma \sim 0.1 \mathrm{Jy} \mathrm{km} / \mathrm{s} \mathrm{beam}^{-1}\right)$. In the right panel, the ivory contours represent the moment-1 in steps of $50 \mathrm{~km} \mathrm{~s}{ }^{-1}$. The black line shows the direction of the major axis. The beam size is shown in the bottom-left corner of each plot.

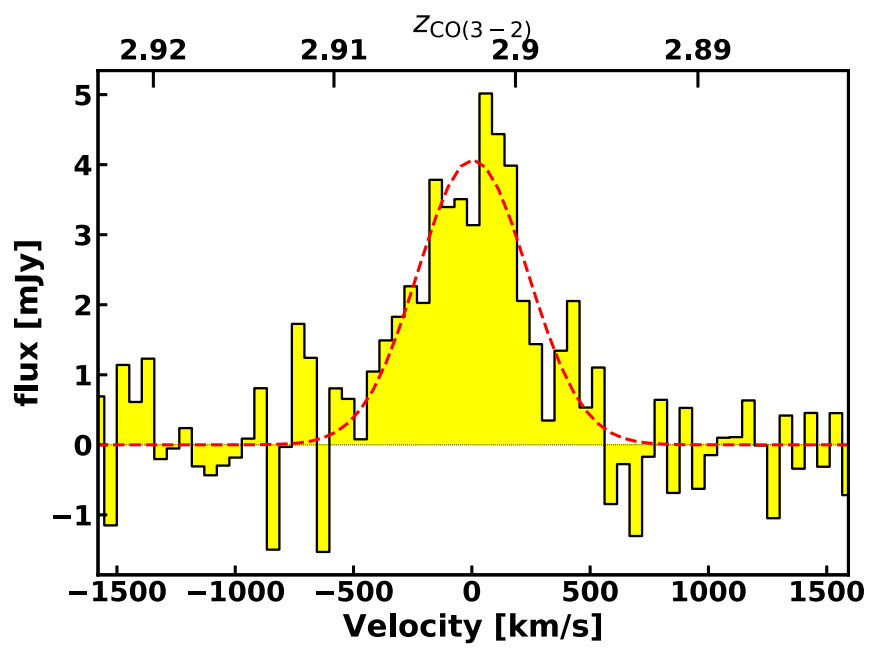

Figure 4. Continuum-subtracted $\mathrm{CO}(3-2)$ spectrum. The red dashed line shows a fit with a Gaussian function.

band, and $B\left(\nu_{\text {rest }}, T_{\text {dust }}\right)$ is the Planck function at temperature $T_{\text {dust }}$. Adopting the best-fit values $T_{\text {dust }}=78.1 \mathrm{~K}, \beta=1.84$, and $\kappa_{1} \mathrm{THz}=20 \mathrm{~cm}^{2} \mathrm{~g}^{-1}$ which is the same as in Wu et al. (2014) and Fan et al. (2016), we derive the dust mass $M_{\text {dust }}=$ $(8.9 \pm 0.5) \times 10^{7} M_{\odot}$. The small uncertainty of dust mass estimation only takes the uncertainties of the derived $T_{\text {dust }}$ and $\beta$ values into account. We note that the largest uncertainty can arise from the adopted $\kappa_{\nu_{\text {rest }}}$ value, which can vary by over one order of magnitude at a certain frequency/wavelength. For instance, $\kappa_{850 \mu \mathrm{m}}$ can vary from $\sim 0.4$ to $\sim 11 \mathrm{~cm}^{2} \mathrm{~g}^{-1}$ in the literature (e.g., James et al. 2002; Draine 2003; Dunne et al. 2003; Siebenmorgen et al. 2014).

We derive the gas-to-dust mass ratio of W0533-3401, $\delta_{\mathrm{GDR}}=944 \pm 77$, based on the estimations of $M_{\mathrm{H}_{2}}$ and $M_{\mathrm{dust}}$. The derived $\delta_{\mathrm{GDR}}$ value of W0533-3401 is thus about one order of magnitude higher than the typical value $\sim 50-150$ derived for the Milky Way (Jenkins 2004), the local starforming galaxies (SFGs) and ultraluminous IR galaxies (ULIRGs) at solar metallicity (Draine et al. 2007; Rémy-Ruyer et al. 2014) and high-redshift SMGs (Magnelli et al. 2012; Miettinen et al. 2017). The high $\delta_{\mathrm{GDR}}$ value in W0533-3401 may be due to several possible reasons: the uncertainty of dust

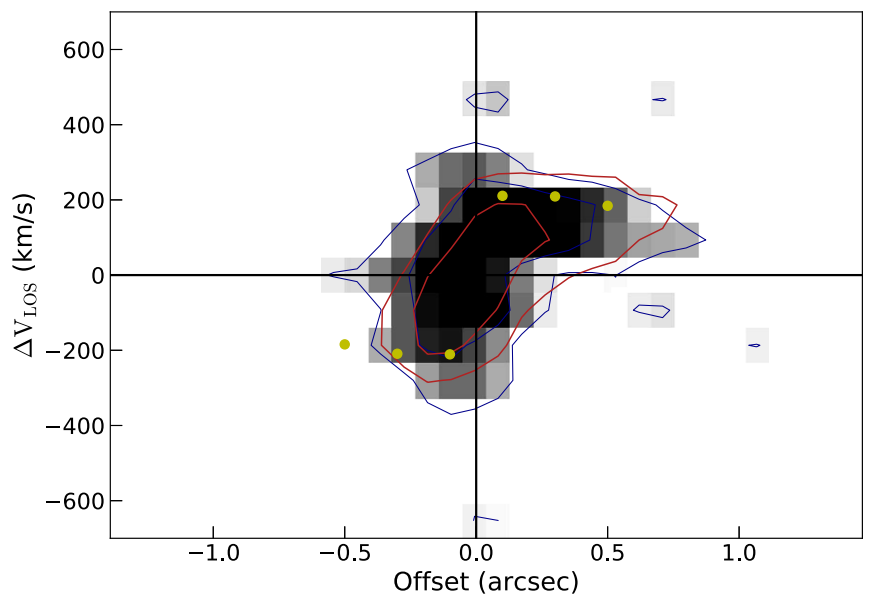

Figure 5. CO (3-2) position vs. velocity (PV) diagram extracted along the major axis at P.A. $=330^{\circ}$ assuming an inclination of $59^{\circ}$. The blue and red contours indicate the isodensity contours of the galaxy and ${ }^{3 \mathrm{D}} \mathrm{BAROLO}$ best-fit model. The yellow points mark the rotation curve with rotation velocities of 246,244 , and $215 \mathrm{~km} \mathrm{~s}^{-1}$ (from center to outer disk), assuming a disk model with three rings, each having a ring width of 0 !' 2 .

mass estimation, the low efficiency of dust formation, and/or the high efficiency of dust destruction. The dust mass derived by using a graybody model is about half of that derived by the Draine \& Li (2007) model (Magdis et al. 2011). This will result in an overestimation of $\delta_{\mathrm{GDR}}$ by a factor of two. The $\delta_{\mathrm{GDR}}$ value is reported to increase with the decreasing metallicity and the increasing redshift (e.g., Rémy-Ruyer et al. 2014; Miettinen et al. 2017). It is possible that W0533-3401 has a low, subsolar metallicity. It is also possible that dust destruction in W0533-3401 may be efficient due to the strong radiation field from massive young stars and AGN, and the supernova shock waves which are expected to be frequent in this maximumstarburst galaxy (Jones 2004).

\subsection{Stellar Mass and Star Formation Rate}

Based on our best-fit SED model presented in Section 3.2, we derive the stellar mass and star formation rate (SFR) of W0533-3401 and list them in Table 4. The stellar mass $M_{\star}$ is derived by adopting an exponentially declining SFH, which is represented by a young stellar population. We check if there is an old stellar population which is omitted by the present SED 


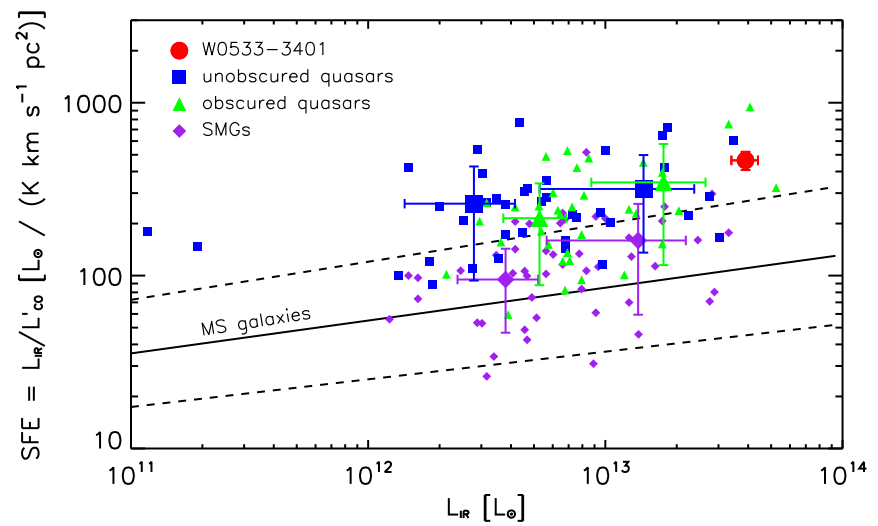

Figure 6. Star formation efficiency (SFE) traced by the ratio of IR to $\mathrm{CO}(1-0)$ line luminosities as a function of IR luminosity. W0533-3401 is labeled with a red circle symbol. The purple diamond, blue square, and green triangle symbols represent the samples of SMGs, unobscured and obscured quasars at $z>1$, respectively, compiled in Perna et al. (2018). Each sample has been divided into two bins according to the IR luminosity. Larger symbols with error bars show the average values and uncertainties for each bin. The solid line and two dashed lines show the best-fit relation and $1 \sigma$ scatter for massive MS galaxies, respectively (Sargent et al. 2014).

fitting procedure. We add an SSP with an age of 1 Gyr to our multiwavelength SED model. The result is that the contribution of the old SSP to the derived stellar mass is not larger than $20 \%$, though the constraint from the SED fitting is loose. Given $M_{\star}=$ $3.5 \times 10^{10} M_{\odot}$ and $M_{\mathrm{H}_{2}}=8.4 \times 10^{10} M_{\odot}$, we can calculate the molecular gas fraction $f_{\mathrm{gas}}=M_{\mathrm{H}_{2}} /\left(M_{\mathrm{H}_{2}}+M_{\star}\right)=0.71$, which indicates that W0533-3401 is gas-rich. The derived SFR is $\sim 7000 M_{\odot} \mathrm{yr}^{-1}$. The specific SFR or $\mathrm{sSFR}=200 \mathrm{Gyr}^{-1}$ of W0533-3401 is over one order of magnitude higher than the star-forming main-sequence (MS) at $z \sim 3$ (Speagle et al. 2014), suggesting that it is a maximum starburst. The uncertainty of SFR is large $\left(\sim 3000 M_{\odot} \mathrm{yr}^{-1}\right)$ due to a wide range of possible SFH. By using $\mathrm{CO}$ line luminosity, we can estimate SFR in an individual way. First, we convert the measured $L_{\mathrm{CO}(3-2)}^{\prime}$ to $L_{\mathrm{CO}(5-4)}^{\prime}$ by taking $L_{\mathrm{CO}(5-4)}^{\prime} / L_{\mathrm{CO}(3-2)}^{\prime}=0.7$ which is intermediate between the typical values for SMGs and quasars (Carilli $\&$ Walter 2013). Then we estimate FIR luminosity using the high$\mathrm{J}$ CO versus FIR luminosity relation presented in Liu et al. (2015). Using the relation SFR $\left(M_{\odot} \mathrm{yr}^{-1}\right)=4.5 \times 10^{-44} L_{\mathrm{FIR}}$ $\left(\mathrm{erg} \mathrm{s}^{-1}\right)$, we calculate $\mathrm{SFR} \sim 3000 M_{\odot} \mathrm{yr}^{-1}$ (Kennicutt 1998), which is generally consistent with the best-fit SED result. From the derived SFR and molecular gas mass, we can estimate the gas depletion timescale $t_{\text {depl }}=M_{\text {gas }} /$ SFR. We infer $t_{\text {depl }} \sim$ 12-28 Myr using SFRs based on the best-fit SED result and the $\mathrm{CO}$ line luminosity, respectively. The gas depletion timescale of W0533-3401 is similar to other obscured quasars (e.g., Aravena et al. 2008; Brusa et al. 2018), but is much shorter than MS galaxies and SMGs (Bothwell et al. 2013; Sargent et al. 2014), indicating that W0533-3401 as an obscured quasar is consuming its residual gas more rapidly.

In Figure 6, we report the star formation efficiency (SFE), traced by the ratio of IR to $\mathrm{CO}(1-0)$ line luminosities, as a function of IR luminosity for W0533-3401 and the compiled samples of SMGs, unobscured and obscured quasars at $z>1$ in Perna et al. (2018). For all three samples, SFE shows a positive correlation with IR luminosity. W0533-3401 shows a high $\mathrm{SFE}=L_{\mathrm{IR}} / L_{\mathrm{CO}}^{\prime}=464 \pm 56 L_{\odot} /\left(\mathrm{K} \mathrm{km} \mathrm{s}^{-1} \mathrm{pc}^{2}\right)$, which is well above the best-fit relation and $1 \sigma$ scatter for massive MS galaxies (Sargent et al. 2014). Similar to W0533-3401, both unobscured and obscured quasars at $z>1$ show a higher SFE at a given IR luminosity than MS galaxies and SMGs. Higher $\mathrm{SFE}$ in unobscured and obscured quasars relative to MS galaxies and SMGs is possibly due to the presence of starburst activity and/or the depletion of cold gas by AGN feedback in quasar host galaxies. The former enhances $L_{\mathrm{IR}}$ and the latter reduces $L_{\mathrm{CO}}^{\prime}$. It is possible that $L_{\mathrm{IR}}$ can be overestimated in quasars, as AGN-heated dust on kiloparsec scales can contribute significantly to IR luminosity (Duras et al. 2017; Symeonidis 2017).

\subsection{Rapid Growth of Both the Stellar Component and the Central SMBH}

We derive the AGN bolometric luminosity $L_{\mathrm{AGN}}$ of W0533 -3401 by integrating the AGN component of the best-fit UVto-millimeter SED (see Section 3.2). Although we do not have a direct measurement of the black hole mass in W0533-3401, we can make a rough estimate from the derived $L_{\mathrm{AGN}}$. By assuming Eddington ratios of 0.3, 1.0, and 3.0, the corresponding black hole masses are $7.3 \times 10^{9}, 2.2 \times 10^{9}$, and $7.3 \times 10^{8} M_{\odot}$, respectively. Wu et al. (2018) reported the black hole masses and Eddington ratios of five luminous obscured quasars at $z \sim 2$, which are taken from the same parent sample of W0533-3401, based on broad $\mathrm{H}_{\alpha}$ lines. They found that the average black hole mass is about $10^{9} M_{\odot}$ and the derived Eddington ratios are close to unity. Tsai et al. (2018) also reported the measurement of $M_{\mathrm{BH}}$ and $L_{\mathrm{Edd}}$ for an extremely luminous, obscured quasar W2246-0526 at $z=4.6$, which is selected with the same criteria as W0533-3401. They found that the central SMBH in it is growing rapidly by accreting at a super-Eddington ratio $\left(\lambda_{\text {Edd }}=2.8\right)$. Adopting the Eddington ratio $\lambda_{\mathrm{Edd}}=1.0$ for $\mathrm{W} 0533-3401$ will be a reasonable approximation.

From SED-based stellar mass $M_{\star}$ and the estimated SMBH mass, we can infer the black hole to stellar mass ratio of W0533 -3401. In Figure 7, we plot the observed $M_{\mathrm{BH}} / M_{\star}$ as a function of redshift for W0533-3401 and several other samples in the literature. If assuming $\lambda_{\text {Edd }}=1.0$, the black hole to stellar mass ratio of W0533-3401 $\left(M_{\mathrm{BH}} / M_{\star}=0.063\right)$ is close to that of an X-ray selected unobscured quasar, CID947, which has the highest $M_{\mathrm{BH}} / M_{\star}(1 / 8)$ at $z \sim 3.3$ known so far. A low Eddington ratio in W0533-3401, for instance $\lambda_{\text {Edd }}<0.3$, seems not likely, otherwise $M_{\mathrm{BH}} / M_{\star}$ of W0533 -3401 will be higher than 0.2 . Even if taking $\lambda_{\text {Edd }}=1.0$, the inferred $M_{\mathrm{BH}} / M_{\star}$ of W0533-3401 is not only over one order of magnitude higher than the typical values $\sim 0.0002-0.0005$ in the local universe (Kormendy \& Ho 2013), but also $\sim 5$ times higher than the expected value by the evolutionary trend of $M_{\mathrm{BH}} / M_{\star}$ (McLure et al. 2006; Peng et al. 2006; Merloni et al. 2010; Targett et al. 2012; Bongiorno et al. 2014; Matsuoka et al. 2018). We derive the black hole mass growth rate $\dot{M}_{\mathrm{BH}}$ of W0533-3401 from the bolometric luminosity by using the relation $L_{\mathrm{AGN}}=\left(\eta \dot{M}_{\mathrm{BH}} c^{2}\right) /(1-\eta)$ and adopting $\frac{\eta}{1-\eta}=0.1$. We infer $\dot{M}_{\mathrm{BH}}=49 M_{\odot} \mathrm{yr}^{-1}$, which suggests a rapid growth of the central SMBH. We then calculate the ratio between the black hole mass growth rate and SFR and obtain $\dot{M}_{\mathrm{BH}} / \mathrm{SFR}=$ 0.007 , which is close to the local $M_{\mathrm{BH}} / M_{\star}$ values. The result indicates that W0533-3401 is evolving toward the evolutionary trend of $M_{\mathrm{BH}} / M_{\star}$ (the dashed line in Figure 7). 




Figure 7. Observed cosmic evolution of black hole to stellar mass ratio $\left(M_{\mathrm{BH}} /\right.$ $\left.M_{\star}\right)$. The $M_{\mathrm{BH}} / M_{\star}$ ratios of W0533-3401 are shown as three star symbols by assuming different Eddington ratios $\lambda_{\text {Edd }}=L_{\text {bol }} / L_{\text {Edd }}=0.3,1.0$, and 3.0, respectively. Other data points represent the available $M_{\mathrm{BH}} / M_{\star}$ estimates in the literature. Color-coded symbols show the average values and the corresponding uncertainties of $M_{\mathrm{BH}} / M_{\star}$ for several samples: blue triangles for a sample of broad-line AGN in the redshift range $1<z<2.2$ (Merloni et al. 2010), brown squares for a sample of lensed and nonlensed quasars at $1<z<4.5$ (Peng et al. 2006), and cyan diamond for a sample of X-ray obscured, red AGN (Bongiorno et al. 2014). Two luminous SDSS quasars at $z \sim 4$ are denoted with purple filled triangles (Targett et al. 2012). The gold filled square shows an extremely red dust-obscured quasar (WISE J1042 +1641 ) at $z=2.52$ (Toba \& Nagao 2016; Matsuoka et al. 2018). The green filled diamond denotes an X-ray selected unobscured quasar, CID-947 at $z \sim 3.3$, which has a high black hole to stellar mass ratio $M_{\mathrm{BH}} / M_{\star}=1 / 8$ (Trakhtenbrot et al. 2015). The dashed line shows the evolutionary trend of $M_{\mathrm{BH}} / M_{\star}$ at $z<2$ (McLure et al. 2006). The gray area shows the range of the typical values of $M_{\mathrm{BH}} / M_{\star}$ in the local universe (Kormendy \& Ho 2013).

\section{Summary and Conclusions}

We present ALMA observations of cold dust and molecular gas and multiwavelength SED analysis in a WISE-selected, hyperluminous dust-obscured quasar W0533-3401 at $z=2.9$. We derive the physical properties of each component, such as molecular gas, stars, dust, and the central SMBH. We summarize our main results as follows.

1. Our ALMA band-3 observations detect both the dust continuum and the $\mathrm{CO}$ (3-2) line. The derived molecular gas mass, $M_{\text {gas }}=8.4 \times 10^{10} M_{\odot}$ and its fraction $f_{\text {gas }}=$ 0.7 suggest that W0533-3401 is gas-rich. Based on the FWHM of the CO (3-2) line, we estimate the dynamical mass $M_{\text {dyn }}=1.6 \times 10^{10} M_{\odot}$, which is generally consistent with the sum of the molecular gas mass and stellar mass. The velocity map of the CO (3-2) emission line showing a velocity gradient is possibly due to a rotating gas disk. However, other possibilities, such as mergers and outflows, cannot be ruled out. Under the assumption of rotation disk, we can roughly estimate the rotation velocity and velocity dispersion. The ratio $V_{\text {rot }} / \sigma$ is large, indicating that the gas disk is possibly unstable, which may be partly responsible for the observed high star formation efficiency in W0533-3401.

2. By assuming a graybody model, we derive the cold dust temperature $T_{\text {dust }}=78.1 \pm 5.6 \mathrm{~K}$ and dust mass $M_{\text {dust }}=(8.9 \pm 1.6) \times 10^{7} M_{\odot}$. The gas-to-dust ratio of $\mathrm{W} 0533-3401, \sigma_{\mathrm{GDR}}=944 \pm 77$, is about one order of magnitude higher than the typical values for the Milky Way, the local SFGs/ULIRGs, and high-redshift SMGs.

3. Based on the UV-to-millimeter SED modeling, we derive the stellar mass and SFR of W0533-3401. The stellar mass of W0533-3401 is $(3.5 \pm 0.9) \times 10^{10} M_{\odot}$.
The SFR is estimated to be $\sim 3000-7000 M_{\odot} \mathrm{yr}^{-1}$ by using different methods. The high values of SFR and specific SFR suggest W0533-3401 is a maximum starburst. The corresponding gas depletion timescales are very short $\left(t_{\text {depl }} \sim 12-28 \mathrm{Myr}\right)$.

4. Finally, we infer the black hole mass growth rate of W0533 -3401 $\left(\dot{M}_{\mathrm{BH}}=49 M_{\odot} \mathrm{yr}^{-1}\right)$, which suggests a rapid growth of the central SMBH. The observed black hole to stellar mass ratio $M_{\mathrm{BH}} / M_{\star}$ of W0533-3401, which is dependent on the adopted Eddington ratio, is over one order of magnitude higher than the local value if assuming a reasonable Eddington ratio $\lambda_{\mathrm{Edd}}=1.0$, and is evolving toward the evolutionary trend of unobscured quasars.

5. Our results suggest that W0533-3401 has both a gas-rich maximum starburst and a rapid black hole growth within it. All results are consistent with the scenario that W0533 -3401 is experiencing a short transition phase toward an unobscured quasar.

We thank the anonymous referee for constructive comments and suggestions. We thank Dr. Hu Zou (NAOC) for his help on optical photometry. This work is supported by the National Key R\&D Program of China (No. 2017YFA0402703). We thank the staff of the Nordic ALMA Regional Center (ARC) node for their support and helpful discussions. The Nordic ARC node is based at Onsala Space Observatory and funded through Swedish Research Council grant No. 2017-00648. L.F. acknowledges the support from the National Natural Science Foundation of China (NSFC, grant Nos. 11822303, 11773020, and 11433005) and Shandong Provincial Natural Science Foundation, China (ZR2017QA001, JQ201801). K.K. acknowledges support from the Knut and Alice Wallenberg Foundation and the Swedish Research Council. Y.H. acknowledges the support from NSFC (grant No. 11773063) and Natural Science Foundation of Yunnan Province (grant No. 2017FB007). Q.-H.T. acknowledges the support from the NSFC (grant No. 11803090).

This paper makes use of the following ALMA data: ADS/ JAO.ALMA\#2017.1.00441.S. ALMA is a partnership of ESO (representing its member states), NSF (USA), and NINS (Japan), together with NRC (Canada), NSC and ASIAA (Taiwan), and KASI (Republic of Korea), in cooperation with the Republic of Chile. The Joint ALMA Observatory is operated by ESO, AUI/NRAO and NAOJ.

This paper makes use of data products from the Wide-field Infrared Survey Explorer, which is a joint project of the University of California, Los Angeles, and the Jet Propulsion Laboratory/California Institute of Technology, funded by the National Aeronautics and Space Administration.

This paper used public archival data from the Dark Energy Survey (DES). Funding for the DES Projects has been provided by the U.S. Department of Energy, the U.S. National Science Foundation, the Ministry of Science and Education of Spain, the Science and Technology Facilities Council of the United Kingdom, the Higher Education Funding Council for England, the National Center for Supercomputing Applications at the University of Illinois at Urbana-Champaign, the Kavli Institute of Cosmological Physics at the University of Chicago, the Center for Cosmology and Astro-Particle Physics at the Ohio State University, the Mitchell Institute for Fundamental Physics and Astronomy at Texas A\&M University, Financiadora de Estudos e Projetos, Fundação Carlos Chagas Filho de Amparo à Pesquisa do Estado do Rio de Janeiro, Conselho Nacional de Desenvolvimento Científico 
e Tecnológico and the Ministério da Ciência, Tecnologia e Inovação, the Deutsche Forschungsgemeinschaft, and the Collaborating Institutions in the Dark Energy Survey. The Collaborating Institutions are Argonne National Laboratory, the University of California at Santa Cruz, the University of Cambridge, Centro de Investigaciones Energéticas, Medioambientales y Tecnológicas-Madrid, the University of Chicago, University College London, the DES-Brazil Consortium, the University of Edinburgh, the Eidgenössische Technische Hochschule (ETH) Zürich, Fermi National Accelerator Laboratory, the University of Illinois at Urbana-Champaign, the Institut de Ciències de l'Espai (IEEC/CSIC), the Institut de Física d'Altes Energies, Lawrence Berkeley National Laboratory, the Ludwig-Maximilians Universität München and the associated Excellence Cluster Universe, the University of Michigan, the National Optical Astronomy Observatory, the University of Nottingham, The Ohio State University, the OzDES Membership Consortium, the University of Pennsylvania, the University of Portsmouth, SLAC National Accelerator Laboratory, Stanford University, the University of Sussex, and Texas A\&M University. Based in part on observations at Cerro Tololo Inter-American Observatory, National Optical Astronomy Observatory, which is operated by the Association of Universities for Research in Astronomy (AURA) under a cooperative agreement with the National Science Foundation.

Facilities: ALMA, Herschel (PACS, SPIRE), WISE, CTIO (DECam).

\section{ORCID iDs}

Lulu Fan (范璐璐) (1) https://orcid.org/0000-0003-4200-4432 Kirsten K. Knudsen (1) https://orcid.org/0000-0002-7821-8873 Yunkun Han (韩云坤) (1) https://orcid.org/0000-00022547-0434

Qing-hua Tan (谈清华) (1) https://orcid.org/0000-00033032-0948

\section{References}

Abbott, T. M. C., Abdalla, F. B., Allam, S., et al. 2018, ApJS, 239, 18 Alexander, D. M., \& Hickox, R. C. 2012, NewAR, 56, 93 Aravena, M., Bertoldi, F., Schinnerer, E., et al. 2008, A\&A, 491, 173 Assef, R. J., Eisenhardt, P. R. M., Stern, D., et al. 2015, ApJ, 804, 27 Assef, R. J., Walton, D. J., Brightman, M., et al. 2016, ApJ, 819, 111 Banerji, M., Carilli, C. L., Jones, G., et al. 2017, MNRAS, 465, 4390 Bongiorno, A., Maiolino, R., Brusa, M., et al. 2014, MNRAS, 443, 2077 Boquien, M., Burgarella, D., Roehlly, Y., et al. 2019, A\&A, 622, A103 Bothwell, M. S., Smail, I., Chapman, S. C., et al. 2013, MNRAS, 429, 3047 Brusa, M., Cresci, G., Daddi, E., et al. 2018, A\&A, 612, A29 Bruzual, G., \& Charlot, S. 2003, MNRAS, 344, 1000 Calzetti, D., Armus, L., Bohlin, R. C., et al. 2000, ApJ, 533, 682 Carilli, C. L., \& Walter, F. 2013, ARA\&A, 51, 105

Chabrier, G. 2003, PASP, 115, 763

Cutri, R. M. 2013, yCat, 2328

da Cunha, E., Charlot, S., \& Elbaz, D. 2008, MNRAS, 388, 1595 Díaz-Santos, T., Assef, R. J., Blain, A. W., et al. 2016, ApJL, 816, L6 Díaz-Santos, T., Assef, R. J., Blain, A. W., et al. 2018, Sci, 362, 1034 Di Teodoro, E. M., \& Fraternali, F. 2015, MNRAS, 451, 3021 Draine, B. T. 2003, ARA\&A, 41, 241

Draine, B. T., Dale, D. A., Bendo, G., et al. 2007, ApJ, 663, 866 Draine, B. T., \& Li, A. 2007, ApJ, 657, 810

Dunne, L., Eales, S., Ivison, R., Morgan, H., \& Edmunds, M. 2003, Natur, 424,285

Duras, F., Bongiorno, A., Piconcelli, E., et al. 2017, A\&A, 604, A67 Eisenhardt, P. R. M., Wu, J., Tsai, C.-W., et al. 2012, ApJ, 755, 173 Fan, L., Gao, Y., Knudsen, K. K., et al. 2018, ApJ, 854, 157

Fan, L., Han, Y., Fang, G., et al. 2016, ApJL, 822, L32

Fan, L., Han, Y., Nikutta, R., Drouart, G., \& Knudsen, K. K. 2016, ApJ, 823, 107
Fan, L., Jones, S. F., Han, Y., \& Knudsen, K. K. 2017, PASP, 129, 124101 Fan, L., Knudsen, K. K., Fogasy, J., et al. 2018, ApJL, 856, L5 Ferrarese, L., \& Ford, H. 2005, SSRv, 116, 523

Feruglio, C., Fiore, F., Carniani, S., et al. 2018, A\&A, 619, A39 Frey, S., Paragi, Z., Gabányi, K. É, et al. 2016, MNRAS, 455, 2058 Granato, G. L., De Zotti, G., Silva, L., et al. 2004, ApJ, 600, 580 Griffin, M. J., Abergel, A., Abreu, A., et al. 2010, A\&A, 518, L3 Han, Y., \& Han, Z. 2012, ApJ, 749, 123

Han, Y., \& Han, Z. 2014, ApJS, 215, 2

Han, Y., \& Han, Z. 2019, ApJS, 240, 3

Hickox, R. C., \& Alexander, D. M. 2018, ARA\&A, 56, 625

Hopkins, P. F., Cox, T. J., Younger, J. D., et al. 2009, ApJ, 691, 1168 Hopkins, P. F., Hernquist, L., Cox, T. J., et al. 2008, ApJS, 175, 356

Hung, C.-L., Rich, J. A., Yuan, T., et al. 2015, ApJ, 803, 62

James, A., Dunne, L., Eales, S., \& Edmunds, M. G. 2002, MNRAS, 335, 753 Jenkins, E. B. 2004, in Canegie Obs. Centennial Symp., Origin and Evolution of the Elements, ed. A. McWilliam \& M. Rauch (Cambridge: Cambridge Univ. Press), 336

Jones, A. P. 2004, in ASP Conf. Ser. 309,Astrophysics of Dust, ed. A. N. Witt, G. Clayton, \& B. T. Draine (San Francisco, CA: ASP), 347

Jones, S. F., Blain, A. W., Assef, R. J., et al. 2017, MNRAS, 469, 4565 Jones, S. F., Blain, A. W., Lonsdale, C., et al. 2015, MNRAS, 448, 3325 Kennicutt, R. C. 1998, ARA\&A, 36, 189

Komatsu, E., Smith, K. M., Dunkley, J., et al. 2011, ApJS, 192, 18

Kormendy, J., \& Ho, L. C. 2013, ARA\&A, 51, 511

Lang, D. 2014, AJ, 147, 108

Leung, T. K. D., Riechers, D. A., \& Pavesi, R. 2017, ApJ, 836, 180

Liu, D., Gao, Y., Isaak, K., et al. 2015, ApJL, 810, L14

Magdis, G. E., Daddi, E., Elbaz, D., et al. 2011, ApJL, 740, L15

Magnelli, B., Lutz, D., Santini, P., et al. 2012, A\&A, 539, A155

Magorrian, J., Tremaine, S., Richstone, D., et al. 1998, AJ, 115, 2285

Matsuoka, K., Toba, Y., Shidatsu, M., et al. 2018, A\&A, 620, L3

McLure, R. J., Jarvis, M. J., Targett, T. A., et al. 2006, MNRAS, 368, 1395

McMullin, J. P., Waters, B., Schiebel, D., Young, W., \& Golap, K. 2007, in ASP Conf. Ser. 376, Astronomical Data Analysis Software and Systems XVI, ed. R. A. Shaw, F. Hill, \& D. J. Bell (San Francisco, CA: ASP), 127 Meisner, A. M., Lang, D., \& Schlegel, D. J. 2017, AJ, 154, 161 Merloni, A., Bongiorno, A., Bolzonella, M., et al. 2010, ApJ, 708, 137 Miettinen, O., Delvecchio, I., Smolčić, V., et al. 2017, A\&A, 606, A17 Nenkova, M., Sirocky, M. M., Ivezić, Ž, \& Elitzur, M. 2008a, ApJ, 685, 147 Nenkova, M., Sirocky, M. M., Nikutta, R., Ivezić, Ž, \& Elitzur, M. 2008b, ApJ, 685,160

Noll, S., Burgarella, D., Giovannoli, E., et al. 2009, A\&A, 507, 1793

Padovani, P., Alexander, D. M., Assef, R. J., et al. 2017, A\&ARv, 25, 2

Peng, C. Y., Impey, C. D., Rix, H.-W., et al. 2006, ApJ, 649, 616

Perna, M., Sargent, M. T., Brusa, M., et al. 2018, A\&A, 619, A90

Piconcelli, E., Vignali, C., Bianchi, S., et al. 2015, A\&A, 574, L9

Pilbratt, G. L., Riedinger, J. R., Passvogel, T., et al. 2010, A\&A, 518, L1

Poglitsch, A., Waelkens, C., Geis, N., et al. 2010, A\&A, 518, L2

Rémy-Ruyer, A., Madden, S. C., Galliano, F., et al. 2014, A\&A, 563, A31

Ricci, C., Assef, R. J., Stern, D., et al. 2017, ApJ, 835, 105

Sanders, D. B., Soifer, B. T., Elias, J. H., et al. 1988, ApJ, 325, 74

Sargent, M. T., Daddi, E., Béthermin, M., et al. 2014, ApJ, 793, 19

Siebenmorgen, R., Voshchinnikov, N. V., \& Bagnulo, S. 2014, A\&A, 561, A82

Solomon, P. M., \& Vanden Bout, P. A. 2005, ARA\&A, 43, 677

Speagle, J. S., Steinhardt, C. L., Capak, P. L., et al. 2014, ApJS, 214, 15

Springel, V., \& Hernquist, L. 2005, ApJL, 622, L9

Stern, D., Lansbury, G. B., Assef, R. J., et al. 2014, ApJ, 794, 102

Symeonidis, M. 2017, MNRAS, 465, 1401

Tacconi, L. J., Neri, R., Genzel, R., et al. 2013, ApJ, 768, 74

Tadaki, K., Iono, D., Yun, M. S., et al. 2018, Natur, 560, 613

Talia, M., Pozzi, F., Vallini, L., et al. 2018, MNRAS, 476, 3956

Targett, T. A., Dunlop, J. S., \& McLure, R. J. 2012, MNRAS, 420, 3621

Toba, Y., \& Nagao, T. 2016, ApJ, 820, 46

Trakhtenbrot, B., Urry, C. M., Civano, F., et al. 2015, Sci, 349, 168

Tsai, C.-W., Eisenhardt, P. R. M., Jun, H. D., et al. 2018, ApJ, 868, 15

Tsai, C.-W., Eisenhardt, P. R. M., Wu, J., et al. 2015, ApJ, 805, 90

Ueda, J., Iono, D., Yun, M. S., et al. 2014, ApJS, 214, 1

Vito, F., Brandt, W. N., Stern, D., et al. 2018, MNRAS, 474, 4528

Wang, R., Wagg, J., Carilli, C. L., et al. 2013, ApJ, 773, 44

Wright, E. L., Eisenhardt, P. R. M., Mainzer, A. K., et al. 2010, AJ, 140, 1868

Wu, J., Bussmann, R. S., Tsai, C.-W., et al. 2014, ApJ, 793, 8

Wu, J., Jun, H. D., Assef, R. J., et al. 2018, ApJ, 852, 96

Wu, J., Tsai, C.-W., Sayers, J., et al. 2012, ApJ, 756, 96

Zappacosta, L., Piconcelli, E., Duras, F., et al. 2018, A\&A, 618, A28 